\title{
Miro, MCU, and calcium: bridging our understanding of mitochondrial movement in axons
}

\author{
Robert F. Niescier ${ }^{1}$, Karen T. Chang ${ }^{2,3}$ and Kyung-Tai Min ${ }^{1}$ * \\ ${ }^{1}$ School of Nano-Bioscience and Chemical Engineering, Ulsan National Institute of Science and Technology, Ulsan, South Korea \\ 2 Zilkha Neurogenetic Institute, University of Southern California, Los Angeles, CA, USA \\ ${ }^{3}$ Department of Cell and Neurobiology, University of Southern California, Los Angeles, CA, USA
}

\author{
Edited by: \\ Rena Li, Roskamp Institute, USA \\ Reviewed by: \\ Alexander K. Murashov, East Carolina \\ University, USA \\ Jiangang Long, Xi'an Jiaotong \\ University, China \\ *Correspondence: \\ Kyung-Tai Min, School of \\ Nano-Bioscience and Chemical \\ Engineering, Ulsan National Institute \\ of Science and Technology, Stem Cell \\ Research Building 105-221, Ulju, \\ Eonyang, Ulsan, South Korea \\ e-mail:ktaimin@unist.ac.kr
}

Neurons are extremely polarized structures with long axons and dendrites, which require proper distribution of mitochondria and maintenance of mitochondrial dynamics for neuronal functions and survival. Indeed, recent studies show that various neurological disorders are linked to mitochondrial transport in neurons. Mitochondrial anterograde transport is believed to deliver metabolic energy to synaptic terminals where energy demands are high, while mitochondrial retrograde transport is required to repair or remove damaged mitochondria in axons. It has been suggested that $\mathrm{Ca}^{2+}$ plays a key role in regulating mitochondrial transport by altering the configuration of mitochondrial protein, miro. However, molecular mechanisms that regulate mitochondrial transport in neurons still are not well characterized. In this review, we will discuss the roles of miro in mitochondrial transport and how the recently identified components of the mitochondrial calcium uniporter add to our current model of mitochondrial mobility regulation.

Keywords: mitochondria, axonal transport, MCU, MICU1, miro

\section{INTRODUCTION}

Mitochondria are vital organelles that provide ATP, maintain $\mathrm{Ca}^{2+}$ homeostasis, and regulate apoptosis in all eukaryotic cells. For cells with high-energy requirement such as neurons, mitochondria are particularly important for neuronal survival, membrane excitability, calcium buffering, and reliable synaptic transmission (Courchet et al., 2013; Obashi and Okabe, 2013; Shao et al., 2013). In addition, the extremely long processes of neurons pose a unique challenge in distributing mitochondria to the appropriate locations; an inability to maintain the dynamics of mitochondrial transport in neurons can thus cause deleterious effects on their function and physiology (Schon and Przedborski, 2011). It has become clear that dysregulation of mitochondrial transport contributes to pathological changes of neurons, as various neurodegenerative diseases such as Parkinson's, Alzheimer's, Huntington's disease, and Down syndrome are associated with defects in mitochondrial transport or dynamics (Schon and Przedborski, 2011). While these neurodegenerative diseases result from different genetic mutations or arise sporadically, the function of the respective gene products may be involved in common pathways leading to altered mitochondrial transport or dynamics in neurons. However, molecular mechanisms that regulate mitochondrial transport in neurons are not well characterized, and how defective mitochondrial transport leads to neurodegeneration remains unclear.

Mitochondrial transport is known to be mediated by interactions between the mitochondrial adaptor proteins to kinesin and dynein motors, as well as the binding of the motor proteins to the cytoskeleton track (Pilling et al., 2006; Russo et al., 2009; reviewed in Saxton and Hollenbeck, 2012). In spite of this now widely held point of view, it still remains unclear whether internal parameters in mitochondria provide another level of regulation that increases the efficiency of mitochondrial transport in axons. It is generally believed that mitochondrial transport in axons is passive, and the internal state of the mitochondrion is inconsequential. In this view, mitochondrial transport is regulated mainly by extracellular signals that modulate cytoplasmic $\mathrm{Ca}^{2+}$ influx, which in turn controls mitochondrial transport machinery (Saotome et al., 2008). It was posited that cytoplasmic $\mathrm{Ca}^{2+}$ level is a key regulator of mitochondrial trafficking in axons and dendrites, and that intracellular $\mathrm{Ca}^{2+}$ influx impedes mitochondrial movement by affecting the overall interactions between the mitochondrial adaptor, motor, and cytoskeleton track. In contrast, we recently discovered that intra-mitochondrial $\mathrm{Ca}^{2+}$ plays a critical role in mitochondrial transport in axons (Chang et al., 2011), suggesting that intrinsic signals inside of mitochondria may be actively involved in mitochondrial transport. The intra-mitochondrial $\mathrm{Ca}^{2+}$ level is mostly regulated by the mitochondrial calcium uniporter (MCU) complex, and three components of the MCU complex have been recently identified: MCU (Baughman et al., 2011; De Stefani et al., 2011), mitochondrial calcium uptakel (MICU1; Perocchi et al., 2010), and mitochondria calcium uniporter regulator 1 (MCUR1; Mallilankaraman et al., 2012a). In this review, we will discuss the new prospects of miro and the MCU complex in regulating mitochondrial transport in axons.

\section{Miro: A MULTIFUNCTION PROTEIN INVOLVED IN REGULATION OF MITOCHONDRIAL TRANSPORT}

Mirol is a mitochondrial outer membrane protein in which the N-terminal part contains two GTPase domains separated by two EF-hand domains facing the cytoplasm (Fransson etal., 2003, 2006). It also contains a single transmembrane domain on the 
C-terminus, with a small three amino acid tail leading into the intermembrane space (Fransson et al., 2006). There are two isoforms of miro, classified as miro1 and miro2, which possess a $60 \%$ similarity (Fransson et al., 2003). Mirol has been shown to play a role in mitochondrial transport in neurons, but a divergent role for miro2, if any exists, has not been explored. Results have shown that overexpression of the two proteins produce slightly different phenotypes on mitochondrial morphology: mirol produces both aggregated and threadlike mitochondria, while miro 2 only generates aggregated mitochondria (Fransson et al., 2006), suggesting that the roles of miro1 and miro2 are slightly different on mitochondrial structure.

Mirol is known as the primary regulator of anterograde mitochondrial movement along microtubules in axons and dendrites through an indirect interaction with kinesin KIF5B (Tanaka et al., 1998). Normally, miro1 binds to TRAK2 (Brickley and Stephenson, 2011; Milton in Drosophila; Glater et al., 2006), a kinesin light chain adapter, which in turn binds directly to kinesin heavy chain and mediates transport along microtubules (Stowers et al., 2002; Glater et al., 2006). However, when cytoplasmic $\mathrm{Ca}^{2+}$ levels increases, $\mathrm{Ca}^{2+}$ binding to the EF-hand domains of mirol results in a conformational shift that subsequently arrests mitochondrial movement (Figure 1, a). The mechanism to explain mirol's role in halting mitochondrial movement is still not clear. Wang and Schwarz (2009) have shown that $\mathrm{Ca}^{2+}$ binding to mirol derails the kinesin motor protein from the microtubule track, thereby stopping mitochondrial transport in axons. In contrast, Macaskill et al. (2009) suggested that high levels of cytoplasmic $\mathrm{Ca}^{2+}$ triggers mirol to dissociate from kinesin motor together with the adaptor protein, TRAK2, thereby arresting mitochondrial transport.

Studies have suggested that the dynein protein is responsible for retrograde movement. Interestingly, evidences suggest that mirol is also required for retrograde movement and that kinesin-1 in Drosophila must be expressed in order for retrograde movement to occur (Pilling et al., 2006). A recent report demonstrated that miro-associated adapter protein TRAK1 binds to both kinesin and dynein components, while TRAK2 preferentially interacts with dynein (van Spronsen et al., 2013). The differential bindings of TRAK proteins to kinesin and dynein seem to play a critical role in targeting of mitochondria to dendrites and axons, as well as in the bi-directional movement of mitochondria in neurons. However, despite this new information, what regulates the switch between retrograde and anterograde movement still remains unknown.

Recently, mirol is found to be a substrate of PINK1 (a kinase) and Parkin (an E3 ubiquitin ligase) in neurons (Wang et al., 2011). PINK1 and Parkin are well-known regulators of mitochondrial health and mitophagy (Narendra etal., 2010), and mutations in these proteins are responsible for familial Parkinson's disease (Kitada et al., 1998; Valente et al., 2001).

Wang et al. (2011) demonstrated that PINK1 phosphorylates miro1, which triggers Parkin-dependent miro degradation, consequently stopping mitochondrial transport in axons (Figure 1, b). Overexpression of PINK1 significantly decreases the frequency of mitochondrial movement in axons. Furthermore, PINK1 and

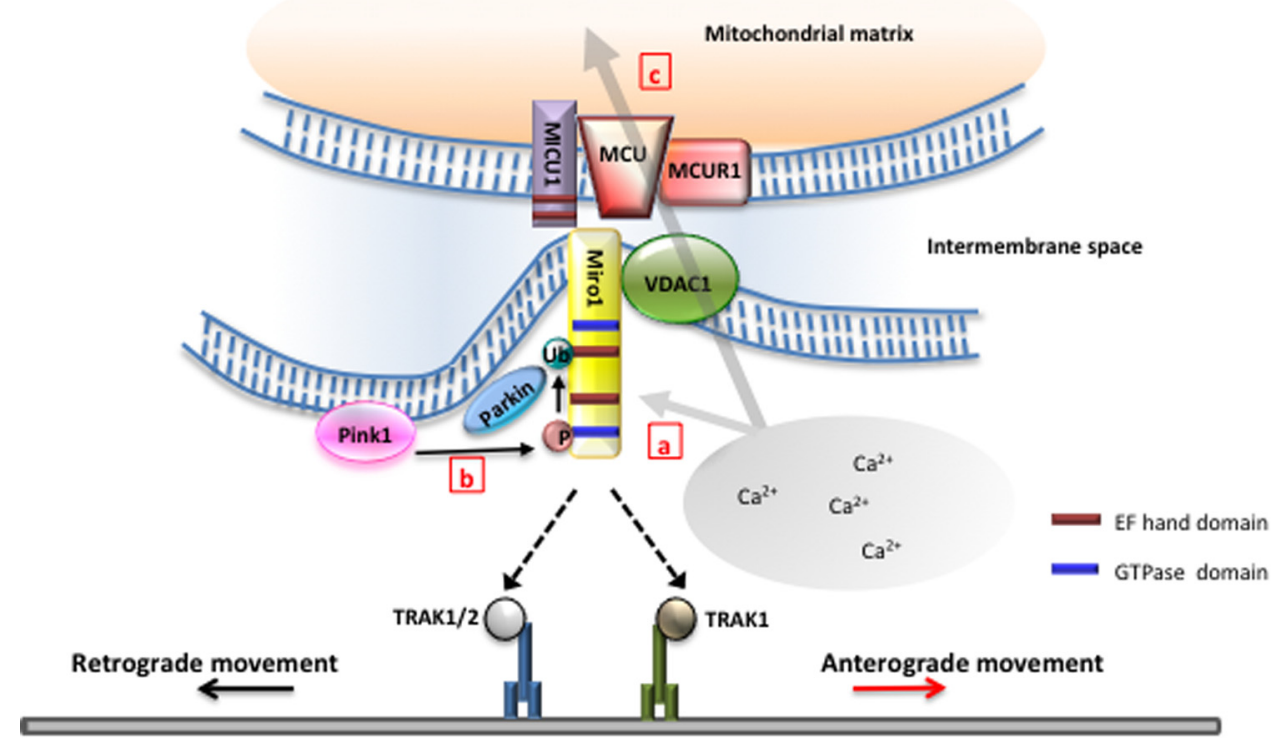

FIGURE 1 | Miro is the nexus of mitochondrial function and transport. (a) Miro1 has been proposed to differentially regulate motor-microtubule interaction Mang and Schwarz, 2009) or TRAK-motor interaction (Macaskill et al., 2009) in the presence of high intracellular $\mathrm{Ca}^{2+}$. Selective binding of miro1 to TRAK1 or TRAK2 may regulate interaction with kinesin or dynein to modulate directionality of mitochondrial movement (van Spronsen etal., 2013). (b) Phosphorylation of miro1 by PINK1 and subsequent ubiquination of miro by parkin have been found to play a critical role in the initial stages of mitophagy Mang etal., 2011). (c) Miro has been found to play a role upstream of the mitochondrial calcium uniporter complex in controlling $\mathrm{Ca}^{2+}$ influx into the matrix. Mutations in the EF-hands of miro1 removes this function (Chang etal., 2011). $\mathrm{Ca}^{2}+$ influx through the mitochondrial calcium uniporter has also been shown to gate mitochondrial movement (Chang etal., 2011). 
Parkin cooperate to remove damaged mitochondria in axons. However, it is to note that mitochondria targeted for degradation did not return to the soma by retrograde movement, but rather remained in axons. While it still remains to be determined if mitophagy indeed occurs in axons, these studies further suggest that mirol is an important player linking altered mitochondrial movement to mitochondrial damage in axons.

In addition to its role as a regulator of mitochondrial transport, mirol also interacts with the endoplasmic reticulum (ER). Mitochondria are known to interact through the ER-mitochondria encounter structures (ERMES; Kornmann et al., 2009), which consist of a variety of proteins that tether the two cellular components together. Gem1, a yeast ortholog of miro1, has been found to be one of the proteins responsible for the connection between ER and mitochondria (Kornmann et al., 2011). Gem1, through one of its EF-hands and the two GTPase domains, regulates the interaction between mitochondria and ER. These findings provide additional complexity to the role of mirol in mitochondrial transport, but raise a possibility that mirol may play a role in regulating $\mathrm{Ca}^{2+}$ influx to mitochondria from the ER.

\section{MITOCHONDRIAL $\mathrm{Ca}^{2+}{ }^{2}$ Miro, AND MITOCHONDRIAL TRANSPORT}

A recent study by Chang et al. (2011) using a genetically encoded green fluorescent protein (GFP)-based fluorescent $\mathrm{Ca}^{2+}$ indicator targeted to the mitochondria, mito-Case12, suggested mitochondrial $\mathrm{Ca}^{2+}$ content is another important parameter regulating mitochondrial transport. Chang et al. (2011) discovered that mitochondrial $\mathrm{Ca}^{2+}$ content correlated inversely with the speed of mitochondrial movement, while no correlation was established for the directionality of mitochondrial transport. Interestingly, this work also offers a new role for mirol in regulating mitochondrial transport through modulation of mitochondrial $\mathrm{Ca}^{2+}$ influx. As mentioned above, previous models proposed that increased cytoplasmic $\mathrm{Ca}^{2+}$ halts mitochondrial movement by binding to miro1 EF-hand domains, thus leading to altered interactions between mirol and the kinesin motor, or the kinesin motor and the microtubule tracks (Macaskill et al., 2009; Wang and Schwarz, 2009). These conclusions were in part derived from studies using the miro EF-hand mutants which failed to halt mitochondrial transport even in the presence of high cytoplasmic $\mathrm{Ca}^{2+}$ environment. In contrast, Chang et al. (2011) found that mutations in the EFhand domain of mirol reduced $\mathrm{Ca}^{2+}$ entry into the mitochondria, which is associated with persistent mitochondrial movement. It is thus plausible that blockage of mitochondrial $\mathrm{Ca}^{2+}$ influx by the mirol EF-hand mutants also contributes to failure in mitochondrial movement arrest in neurons. These results implicate a potential role for mirol in regulating mitochondrial movement through regulation of intra-mitochondrial $\mathrm{Ca}^{2+}$ content, and further imply that intra-mitochondrial $\mathrm{Ca}^{2+}$ is an important determinant of mitochondrial transport. It is likely that there is a critical $\mathrm{Ca}^{2+}$ threshold required for pausing mitochondrial movement.

\section{MCU, MICU1, AND MCUR1: OLD AND NEW PLAYERS IN $\mathrm{Ca}^{2+}$ INFLUX TO MITOCHONDRIA}

Aside from ATP production, mitochondria are important for $\mathrm{Ca}^{2+}$ buffering within cells. $\mathrm{Ca}^{2+}$ influx into mitochondria can stimulate ATP production by stimulating enzymes involved in the tricarboxylic acid (TCA) cycle, the electron transport chain, and ATP synthase complex (Wan et al., 1989). Mitochondrial $\mathrm{Ca}^{2+}$ buffering allows cells to maintain homeostatic cytoplasmic $\mathrm{Ca}^{2+}$ level, and under normal conditions $\mathrm{Ca}^{2+}$ enters the mitochondrial matrix through the MCU complex (Baughman et al., 2011; De Stefani et al., 2011). To further test if $\mathrm{Ca}^{2+}$ content in mitochondrial matrix regulates mitochondrial transport, Chang et al. (2011) used drugs that inhibit or activate the MCU complex and investigated the mobility of mitochondria. They found that blocking MCU complex in the presence of high cytoplasmic $\mathrm{Ca}^{2+}$ preserved mitochondrial movement, suggesting that $\mathrm{Ca}^{2+}$ influx into the mitochondrial matrix plays an obligatory role for mitochondrial movement arrest (Figure 1, c). Furthermore, these results identify the MCU complex as a novel component regulating and gating mitochondrial transport. However, mechanisms by which mitochondrial $\mathrm{Ca}^{2+}$ elevation leads to mitochondrial movement arrest remains to be addressed.

The role and function of the MCU complex were proposed in the 1960s and its properties have been extensively studied, but its molecular nature was only recently identified. Perocchi et al. (2010) first exploited the Mitocarta database of mitochondrial genes in order to search for candidates of the MCU complex in silico, then used a targeted RNAi screen for verification. The criteria for the uniporter protein were to have at least one transmembrane domain, a calcium binding motif such as EF-hands, and universal tissue expression. Subsequently, CBARA1, later renamed as MICU1, was identified a candidate protein that met the criteria (Perocchi et al., 2010). Cells having reduced MICU1 by shRNA showed that $\mathrm{Ca}^{2+}$ influx to mitochondria is prevented even after histamine stimulation that triggers mitochondrial $\mathrm{Ca}^{2+}$ uptake (Perocchi et al., 2010). Furthermore, MICU1 reduction resulted in an uncoupling of $\mathrm{Ca}^{2+}$ from mitochondrial energy metabolism without affecting cell survival (Perocchi et al., 2010). However, despite MICU1 having shown many characteristics of MCU, it is still not a channel protein, but a regulator of the uniporter.

Immediately after the identification of MICU1, two independent works discovered the true calcium uniporter that was subsequently named as the MCU. This channel is sensitive to ruthenium red, located in the inner mitochondrial membrane, has calcium channel activity, and interacts with MICU1 (Baughman et al., 2011; De Stefani et al., 2011). MCU contains a novel motif called DIME that consists of Asp-Ile-Met-Glu located between the two transmembrane domains (Baughman et al., 2011; De Stefani et al., 2011). Mutational analysis revealed that these four amino acid residues play critical roles for $\mathrm{Ca}^{2+}$ influx (Baughman et al., 2011). De Stefani etal. (2011) proposed the MCU topology in which DIME motif faces matrix and two amino termini are present in intermembrane space. On the contrary, other study indicates that the DIME motif is in intermembrane space, while the two protein termini are in matrix (Baughman et al., 2011; De Stefani et al., 2011). This conflicting result should be clarified in future work, but despite the disparity, it is now clear that MCU controls $\mathrm{Ca}^{2+}$ influx into mitochondria.

Perocchi et al. (2010) showed that histamine-induced calcium influx into the mitochondrial matrix is correlated to the level of MICU1. A recent study, however, failed to reproduce the 
result (Mallilankaraman et al., 2012b). Instead, Mallilankaraman et al. (2012b) demonstrated that MICU1 regulates MCU-mediated $\mathrm{Ca}^{2+}$ influx. The authors also identified another regulator of MCU, which is called MCUR1 that binds to MCU and regulates MCU-dependent mitochondrial calcium influx (Mallilankaraman et al., 2012b). It colocalizes with MCU and MICU1 on the inner mitochondrial membrane. Knockdown of MCUR1 causes a decrease in oxidative phosphorylation and calcium uptake, which results in impairment of mitochondrial function (Mallilankaraman et al., 2012a). It is to note that MCU migrates at approximately $450 \mathrm{kDa}$ on native polyacrylamide gel electrophoresis (PAGE) analysis despite of its own molecular weight being about $40 \mathrm{kDa}$ (Baughman et al., 2011). In addition, molecular weight of MICU1 is around $54 \mathrm{kDa}$ (Perocchi et al., 2010) and MCUR1 is $40 \mathrm{kDa}$ (Baughman et al., 2011; De Stefani et al., 2011), respectively. Taken together, this implies that MCU associated with MICU1 and MCUR1 is probably a multi-complex channel with a possibility that more unknown components of MCU machinery have yet to be discovered.

With the MCU complex located in the inner mitochondrial membrane, questions remain as to how $\mathrm{Ca}^{2+}$ influx into the mitochondrial matrix gates mitochondrial movement. The identification of additional components of the MCU complex will certainly help to delineate the cellular mechanisms. It is plausible that component(s) of the MCU complex is also associated with mirol, and the whole complex modulates $\mathrm{Ca}^{2+}$ entry into

\section{REFERENCES}

Baughman, J. M., Perocchi, F., Girgis, H. S., Plovanich, M., BelcherTimme, C. A., Sancak, Y., et al. (2011). Integrative genomics identifies MCU as an essential component of the mitochondrial calcium uniporter. Nature 476, 341-345. doi: 10.1038 /nature 10234

Brickley, K., and Stephenson, F. A. (2011). Trafficking kinesin protein (TRAK)-mediated transport of mitochondria in axons of hippocampal neurons. J. Biol. Chem. 286, 18079-18092. doi: 10.1074/jbc.M111.236018

Chang, K. T., Niescier, R. F., and Min, K.-T. (2011). Mitochondrial matrix $\mathrm{Ca}^{2+}$ as an intrinsic signal regulating mitochondrial motility in axons. Proc. Natl. Acad. Sci. U.S.A. 108, 15456-15461. doi: 10.1073/pnas.1106862108

Courchet, J., Lewis, T. L. Jr., Lee, S., Courchet, V., Liou, D. Y., Aizawa, S., etal. (2013). Terminal axon branching is regulated by the LKB1NUAK1 kinase pathway via presynaptic mitochondrial capture. Cell 153, 1510-1525. doi: 10.1016/j.cell.2013. 05.021

De Stefani, D., Raffaello, A., Teardo, E., Szabò, I., and Rizzuto, R. (2011). A forty-kilodalton protein of the inner membrane is the mitochondrial calcium uniporter. Nature 476, 336340. doi: 10.1038/nature 10230

Fransson, A., Ruusala, A., and Aspenstrom, P. (2003). Atypical rho GTPases have roles in mitochondrial homeostasis and apoptosis. J. Biol. Chem. 278, 6495-6502. doi: 10.1074/jbc.M208609200

Fransson, A., Ruusala, A., and Aspenstrom, P. (2006). The atypical rho GTPases miro-1 and miro-2 have essential roles in mitochondrial trafficking. Biochem. Biophys. Res. Commun. 344, 500-510. doi: 10.1016/j.bbrc.2006.03.163

Glater, E. E., Megeath, L. J., Stowers, R. S., and Schwarz, T. L. (2006). Axonal transport of mitochondria requires milton to recruit kinesin heavy chain and is light chain independent. J. Cell Biol. 173, 545-557. doi: 10.1083/jcb.2006 01067

Kitada, T., Asakawa, S., Hattori, N., Matsumine, H., Yamamura, Y., Minoshima, S., et al. (1998). Mutations in the parkin gene cause autosomal recessive juvenile parkinsonism. Nature 392, 605-608. doi: $10.1038 / 33416$

Kornmann, B., Currie, E., Collins, S. R., Schuldiner, M., Nunnari, J., Weissman, J. S., et al. (2009). An ER-mitochondria tethering complex revealed by a synthetic biology

mitochondria as well as mitochondrial transport in axons. $\mathrm{Ca}^{2+}$ influx through the MCU complex may lead to conformational change in the mirol protein, subsequently exposing the EF-hand domain for $\mathrm{Ca}^{2+}$ binding and causing either detachment of mitochondria from the motor or of the motor from the microtubule track. It will be interesting to determine in the future whether mirol binds to MCU or another component of the uniporter complex.

\section{CONCLUSION}

The MCU complex contains three distinct proteins: MCU, MICU1, and MCUR1, which regulates the influx of calcium into the mitochondrial matrix. It will be particularly interesting to test if manipulation of the identified MCU components can influence mitochondrial transport in the future. In addition, it is possible that miro, which can connect to the ER and regulate mitochondrial movement, would be another component of the MCU complex. Future works involving identification of other MCU components will lead to a better understanding of mechanisms regulating mitochondrial transport in axons.

\section{ACKNOWLEDGMENTS}

The laboratory of Kyung-Tai Min is supported by grants from Ulsan National Institute of Science and Technology, Korea Ministry of Education, Science and Technology, and Fondation Jérôme Lejeune.

screen. Science 325, 477-481. doi: 10.1126/science. 1175088

Kornmann, B., Osman, C., and Walter, P. (2011). The conserved GTPase Geml regulates endoplasmic reticulum-mitochondria connections. Proc. Natl. Acad. Sci. U.S.A. 108, 14151-14156. doi: 10.1073/pnas.1111314108

Macaskill, A. F., Rinholm, J. E., Twelvetrees, A. E., Arancibia-Carcamo, I. L., Muir, J., Fransson, A., et al. (2009). Mirol is a calcium sensor for glutamate receptor-dependent localization of mitochondria at synapses. Neuron 61, 541-555. doi: 10.1016/j.neuron.2009.01.030

Mallilankaraman, K., Cardenas, C., Doonan, P. J., Chandramoorthy, H. C., Irrinki, K. M., Golenar, T., etal. (2012a). MCUR1 is an essential component of mitochondrial $\mathrm{Ca}^{2+}$ uptake that regulates cellular metabolism. Nat. Cell Biol. 14, 1336-1343. doi: 10.1038/nc b2622

Mallilankaraman, K., Doonan, P. Cardenas, C., Chandramoorthy, H. C., Muller, M., Miller, R., et al. (2012b). MICU1 is an essential gatekeeper for MCU-mediated mitochondrial $\mathrm{Ca}^{(2+)}$ uptake that regulates cell survival. Cell 151, 630-644. doi: 10.1016/j.cell.2012. 10.011
Narendra, D. P., Jin, S. M., Tanaka, A. Suen, D. F., Gautier, C. A., Shen, J., etal. (2010). PINK1 is selectively stabilized on impaired mitochondria to activate parkin. PLoS Biol. 8:e1000298. doi: 10.1371/journal.pbio. 1000298

Obashi, K., and Okabe, S. (2013). Regulation of mitochondrial dynamics and distribution by synapse position and neuronal activity in the axon. Eur. J. Neurosci. 38, 2350-2363. doi: 10.1111/ejn.12263

Perocchi, F., Gohil, V. M., Girgis, H. S., Bao, X. R., McCombs, J. E., Palmer, A. E., et al. (2010). MICU1 encodes a mitochondrial EF hand protein required for $\mathrm{Ca}^{(2+)}$ uptake. Nature 467, 291-296. doi: 10.1038/nature09358

Pilling, A. D., Horiuchi, D., Lively, C. M., and Saxton, W. M. (2006). Kinesin- 1 and dynein are the primary motors for fast transport of mitochondria in Drosophila motor axons. Mol. Biol. Cell 17, 2057-2068. doi: 10.1091/mbc.E05-06-0526

Russo, G. J., Louie, K., Wellington, A., Macleod, G. T., Hu, F., Panchumarthi, S., etal. (2009). Drosophila miro is required for both anterograde and retrograde axonal mitochondrial transport. $J$. Neurosci. 29, 5443-5455. doi: 10.1523/JNEUROSCI.5417-08.2009 
Saotome, M., Safiulina, D., Szabadkai, G., Das, S., Fransson, A., Aspenstrom, P., et al. (2008). Bidirectional $\mathrm{Ca}^{2+}$-dependent control of mitochondrial dynamics by the miro GTPase. Proc. Natl. Acad. Sci. U.S.A. 105, 20728-20733. doi: 10.1073/pnas.0808953105

Saxton, W. M., and Hollenbeck, P. J. (2012). The axonal transport of mitochondria. J. Cell Sci. 125, 2095-2104. doi: 10.1242/jcs.053850

Schon, E. A., and Przedborski, S. (2011). Mitochondria: the next (neurode)generation. Neuron 70, 1033-1053. doi: 10.1016/j.neuron. 2011.06.003

Shao, C. Y., Zhu, J., Xie, Y. J., Wang, Z., Wang, Y. N., Wang, Y., et al. (2013). Distinct functions of nuclear distribution proteins LIS1, Ndel1 and NudCL in regulating axonal mitochondrial transport. Traffic 14, 785797. doi: 10.1111/tra.12070

Stowers, R. S., Megeath, L. J., GorskaAndrzejak, J., Meinertzhagen, I. A., and Schwarz, T. L. (2002).
Axonal transport of mitochondria to synapses depends on milton, a novel Drosophila protein. Neuron 36, 1063-1077. doi: 10.1016/S08966273(02)01094-2

Tanaka, Y., Kanai, Y., Okada, Y., Nonaka, S., Takeda, S., Harada, A., et al. (1998). Targeted disruption of mouse conventional kinesin heavy chain, kif5B, results in abnormal perinuclear clustering of mitochondria. Cell 93, 1147-1158. doi: 10.1016/S00928674(00)81459-2

Valente, E. M., Bentivoglio, A. R., Dixon, P. H., Ferraris, A., Ialongo, T., Frontali, M., et al. (2001). Localization of a novel locus for autosomal recessive early-onset parkinsonism, PARK6, on human chromosome 1p35-p36. Am. J. Hum. Genet. 68, 895-900. doi: 10.1086/ 319522

van Spronsen, M., Mikhaylova, M., Lipka, J., Schlager, M. A., van den Heuvel, D. J., Kuijpers, M., et al. (2013). TRAK/milton motor-adaptor proteins steer mitochondrial trafficking to axons and dendrites. Neuron 77, 485502. doi: 10.1016/j.neuron. 2012 . 11.027

Wan, B., LaNoue, K. F., Cheung, J. Y., and Scaduto, R. C. Jr. (1989). Regulation of citric acid cycle by calcium. $J$. Biol. Chem. 264, 13430-13439.

Wang, X., and Schwarz, T. L. (2009). The mechanism of $\mathrm{Ca}^{2+}$-dependent regulation of kinesin-mediated mitochondrial motility. Cell 136, 163-174. doi: 10.1016/j.cell.2008. 11.046

Wang, X., Winter, D., Ashrafi, G., Schlehe, J., Wong, Y. L., Selkoe, D., etal. (2011). PINK1 and parkin target miro for phosphorylation and degradation to arrest mitochondrial motility. Cell 147, 893-906. doi: 10.1016/j.cell.2011. 10.018

Conflict of Interest Statement: The authors declare that the research was conducted in the absence of any commercial or financial relationships that could be construed as a potential conflict of interest.

Received: 28 June 2013; accepted: 22 August 2013; published online: 10 September 2013.

Citation: Niescier RF, Chang KT and Min $K-T$ (2013) Miro, MCU, and calcium: bridging our understanding of mitochondrial movement in axons. Front. Cell. Neurosci. 7:148. doi: 10.3389/fncel.2013. 00148

This article was submitted to the journal Frontiers in Cellular Neuroscience.

Copyright (C) 2013 Niescier, Chang and Min. This is an open-access article distributed under the terms of the Creative Commons Attribution License (CC BY). The use, distribution or reproduction in other forums is permitted, provided the original author(s) or licensor are credited and that the original publication in this journal is cited, in accordance with accepted academic practice. No use, distribution or reproduction is permitted which does not comply with these terms. 